\title{
Assessment of long-term safety and efficacy of intranasal mesenchymal stem cell treatment for neonatal brain injury in the mouse
}

\author{
Vanessa Donega' ${ }^{1}$ Cora H. Nijboer ${ }^{1}$, Cindy T.J. van Velthoven', Sameh A. Youssef ${ }^{2}$, Alain de Bruin², Frank van Bel ${ }^{3}$, \\ Annemieke Kavelaars ${ }^{4}$ and Cobi J. Heijnen ${ }^{1,4}$
}

BACKGROUND: For clinical translation, we assessed whether intranasal mesenchymal stem cell (MSC) treatment after hypoxia-ischemia $(\mathrm{HI})$ induces neoplasia in the brain or periphery at 14 mo. Furthermore, the long-term effects of MSCs on behavior and lesion size were determined.

METHOD: HI was induced in 9-d-old mice. Pups received an intranasal administration of $0.5 \times 10^{6} \mathrm{MSC}$ or vehicle at $10 \mathrm{~d}$ post-HI. Full macroscopical and microscopical pathological analysis of 39 organs per mouse was performed. Sensorimotor behavior was assessed in the cylinder-rearing test at $10 \mathrm{~d}, 28$ d, 6 mo, and 9 mo. Cognition was measured with the novel object recognition test at 3 and 14 mo post-HI. Lesion size was determined by analyzing mouse-anti-microtubule-associated protein 2 (MAP2) and mouse-anti-myelin basic protein (MBP) staining at $5 \mathrm{wk}$ and $14 \mathrm{mo}$.

RESULTS: At 14 mo post-HI, we did not observe any neoplasia in the nasal turbinates, brain, or other organs of $\mathrm{HI}$ mice treated with MSCs. Furthermore, our results show that MSC-induced improvement of sensorimotor and cognitive function is long lasting. In contrast, HI-vehicle mice showed severe behavioral impairment. Recovery of MAP2- and MBP-positive area lasted up to 14 mo following MSC treatment.

CONCLUSION: Our results provide strong evidence of the long-term safety and positive effects of MSC treatment following neonatal $\mathrm{HI}$ in mice.

N eonatal encephalopathy due to hypoxia-ischemia (HI) remains a major health issue worldwide. A HI insult often results in brain damage with subsequent development of motor and cognitive impairments like cerebral palsy and mental retardation (1-5). Currently, hypothermia is the only available treatment, but this treatment is only moderately neuroprotective and solely effective in babies born at term if applied within $6 \mathrm{~h}$ post-HI. Hence, there is a strong need for novel treatment strategies (1-5).
Over the past years, mesenchymal stem cells (MSCs) have become a potentially attractive therapeutic option for several diseases $(6,7)$. The growing interest in MSC therapy is partially due to the immunosuppressive capacities of MSCs. Furthermore, MSCs can be easily and effectively obtained from, e.g., bone marrow or Wharton's jelly and are hardly immunogenic as they do not express MHC class II antigens or costimulatory proteins (e.g., CD40, CD80, and CD86) $(8,9,10)$.

We have recently demonstrated that intranasal MSC treatment significantly improves cognitive and motor behavior and reduces infarct size following neonatal $\mathrm{HI}$ brain injury in the mouse (11). Furthermore, we showed that MSC treatment has a long therapeutic window of $10 \mathrm{~d}$. We demonstrated that intranasally administered MSCs reach the lesion site within $2 \mathrm{~h}$ after application to both nostrils (12). A recent study by our group shows that MSCs promote lesion repair by stimulating endogenous regenerative processes (12) and do not engraft in the brain (13). Moreover, previous studies from our group showed that intracranial MSC treatment increases gene expression of several neurotrophic and anti-inflammatory factors in the brain (13). Therefore, intranasal MSC administration holds strong potential as a noninvasive therapeutic strategy to repair neonatal $\mathrm{HI}$ brain injury.

However, the long-term safety of MSC administration remains under debate, and a longitudinal study that assesses long-term safety and efficacy is still lacking. This is an important question that has to be thoroughly investigated before translation to the clinic is possible. Therefore, we investigated in depth whether MSC treatment is associated with malignancies in the brain or other organs after intranasal MSC treatment at 14 mo post-HI. To this end, mice were submitted to systematic histopathological analysis performed by board-certified mouse pathologists. Furthermore, we determined at 14 mo post-HI whether MSC treatment has effects on sensorimotor and cognitive behavior and the cerebral lesion. 


\section{RESULTS}

MSC Administration Induces Long-Term Motor and Cognitive Improvement Post-HI

To investigate the long-term effects of intranasal MSC treatment on motor performance, we assessed sensorimotor

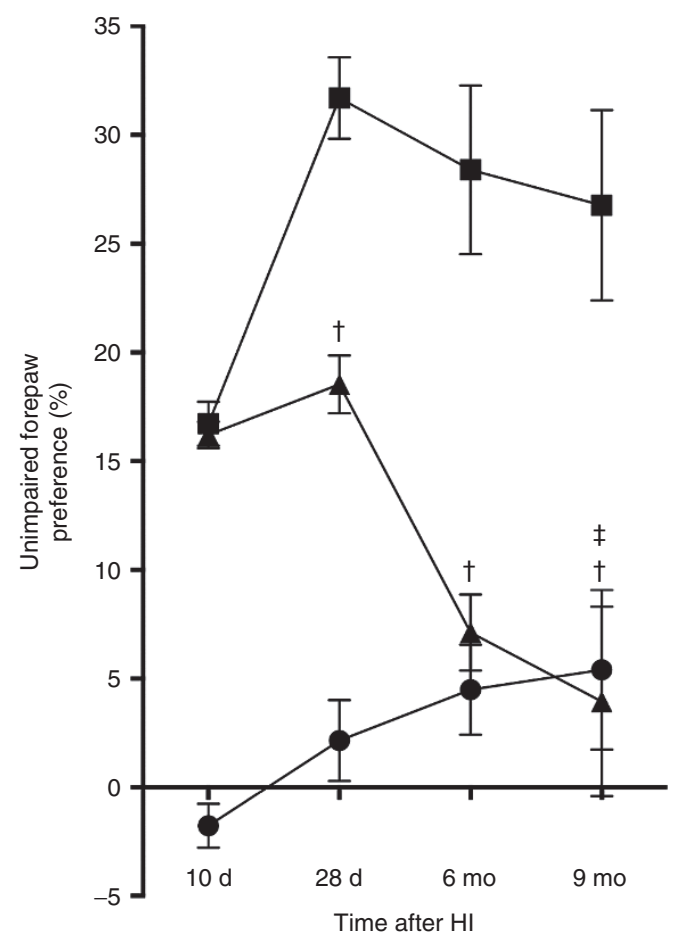

Figure 1. Long-term effect of MSC treatment on sensorimotor behavior over time. Mice received either $0.5 \times 10^{6} \mathrm{MSCs}$ or vehicle intranasally at 10 $\mathrm{d}$ post-HI. Performance in the CRT was assessed at $10 \mathrm{~d}$ (i.e., before treatment) and $28 \mathrm{~d}$ and at 6 and 9 mo post-HI induction or sham operation. The results show that MSC administration improves performance in the CRT up to 9 mo post-HI. ${ }^{\dagger} P<0.001{ }^{\ddagger}{ }^{p}<0.001$ (HI-MSC 28 d vs. HI-MSC 9 mo) with two-way ANOVA and Bonferroni post-hoc test. Animal numbers: 10-d sham, $n=16$; HI-vehicle, $n=17$; HI-MSC, $n=17$; 28-d sham, $n=15$; HI-vehicle, $n=17$; HI-MSC, $n=17$; 6-mo sham, $n=15$; HI-vehicle, $n=17$; HI-MSC, $n=14$; 9-mo sham, $n=13$; HI-vehicle, $n=16$; HI-MSC, $n=13$. HI, hypoxia-ischemia; MSC, mesenchymal stem cell. behavior in the cylinder-rearing test (CRT), which measures the preference to use the unimpaired forepaw during vertical exploration. CRT was measured at 10 and $28 \mathrm{~d}$ and at 6 and 9 mo following sham operation or $\mathrm{HI}$ induction. All $\mathrm{HI}$ mice show similar motor impairment at $10 \mathrm{~d}$ post-HI, i.e., before treatment with MSCs. Our results show that motor impairment in $\mathrm{HI}$-vehicle mice further deteriorated over time. In contrast, MSC treatment at $10 \mathrm{~d}$ post-HI induced long-term improvement of sensorimotor behavior (Figure 1). Our results show a significant decrease in the preference to use the unimpaired forepaw in HI-MSC mice at $28 \mathrm{~d}$ following the insult. At 9 mo post-HI, motor performance of HI-MSC mice had improved even further and impairment had decreased another 79\%, reaching sham level. The total number of full rears in the CRT was similar between all experimental groups at all time points (Supplementary Figure S1 online).

We used the novel object recognition test (NORT) to assess cognitive behavior at 3 and 14 mo after HI. As expected, sham-operated animals showed a significant preference for the novel object at both time points. HI-vehicle mice had no preference for either the novel or the familiar object at 3 or 14 mo after induction of HI (Figure 2a). However, MSC treatment significantly restored cognitive performance in the NORT as our results demonstrate that HI-MSC mice have a significant preference to explore the novel object at both 3 and 14 mo post-HI. There were no differences in total exploration time of the objects between experimental groups at both time points. For instance, sham-operated animals spent $34.79 \pm 3.34 \%$, HI-vehicle $35.73 \pm 3.69 \%$, and HI-MSC $33.32 \pm 3.96 \%$ of time with the objects at 3 mo postHI (Figure 2b)

\section{Changes in Gray and White Matter Damage Over Time}

To assess long-term effects of MSC treatment on the cerebral lesion, we sacrificed mice at $5 \mathrm{wk}$ and 14 mo post-HI or sham operation and analyzed brain sections for loss of MAP2 and MBP staining as a measure of gray- and white-matter damage,
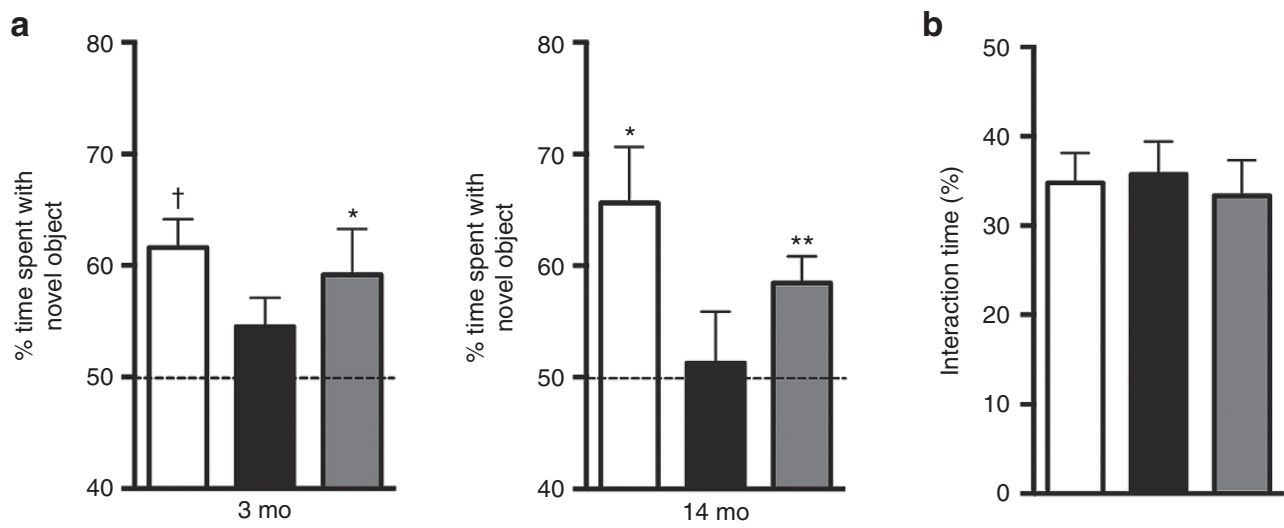

Figure 2. Long-term effect of MSC-treatment on cognitive behavior over time. (a) Cognitive performance in the NORT was assessed at 3 and 14 mo after $\mathrm{HI}$ induction or sham operation. Intranasal MSC administration at $10 \mathrm{~d}$ post-HI significantly improves performance in the NORT up to 14 mo post-HI. (b) Total interaction time with the objects during novel object recognition in all experimental groups at 3 mo. Sham = white bar; vehicle-treated = black bar; MSC-treated = gray bar. ${ }^{*} P<0.05 ;{ }^{*} P<0.01 ;{ }^{\dagger} P<0.001$ with one-sample $t$-test (two-tailed) vs. $50 \%$ interaction time. Three-mo sham, $n=13 ; \mathrm{HI}$-vehicle, $n$ $=15$; HI-MSC, $n=16 ; 14$-mo sham $n=8$; HI-vehicle, $n=10$; HI-MSC, $n=11$. HI, hypoxia-ischemia; MSC, mesenchymal stem cell. 


\section{Articles | Donega et al.}

a

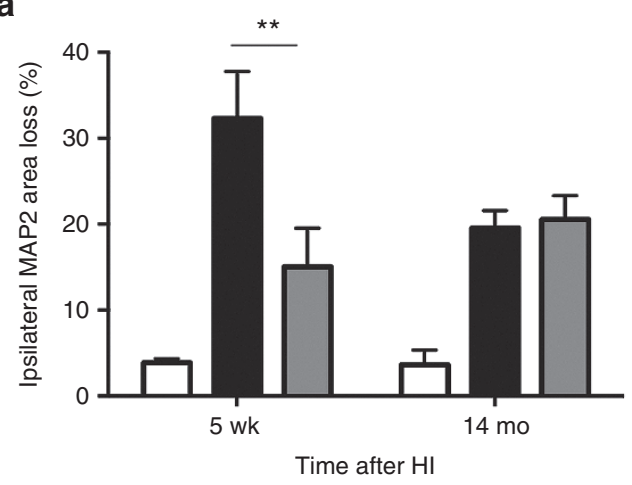

b

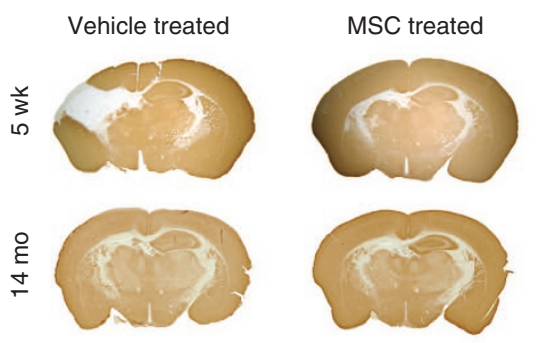

C

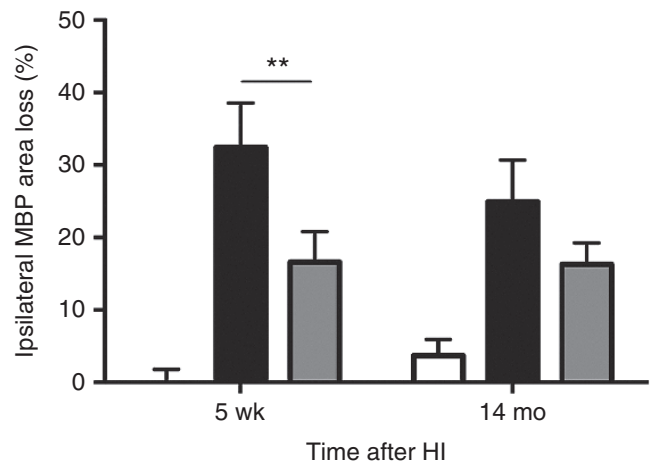

d

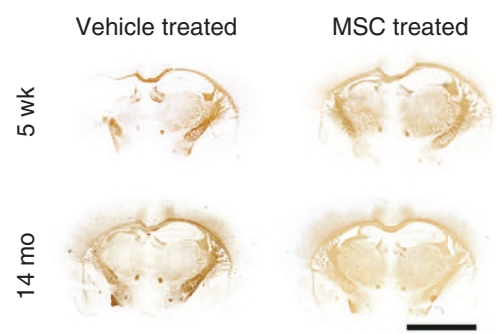

Figure 3. Long-term effect of MSCs on gray and white matter injury over time. Ipsilateral loss of MAP2 and MBP at 5 wk and 14 mo post-HI. (a) MSC treatment significantly decreases MAP2 area loss at $5 \mathrm{wk}$ post-HI. (b) Representative sections of MAP2 staining. (c) MSC treatment decreases MBP area loss at 5 wk post-HI. (d) Representative sections of MBP staining. Sham $=$ white bar; vehicle-treated $=$ black bar; MSC-treated $=$ gray bar. ${ }^{* *} P<0.01$ with one-way ANOVA and Bonferroni post-hoc test. MAP2/MBP 5 wk: sham, $n=3$; HI-vehicle, $n=9$; HI-MSC, $n=10$; MAP2/MBP 14 mo: sham, $n=3$; HI-vehicle, $n=7$; HI-MSC, $n=7$. Bar $=5 \mathrm{~mm}$. HI, hypoxia-ischemia; MSC, mesenchymal stem cell.

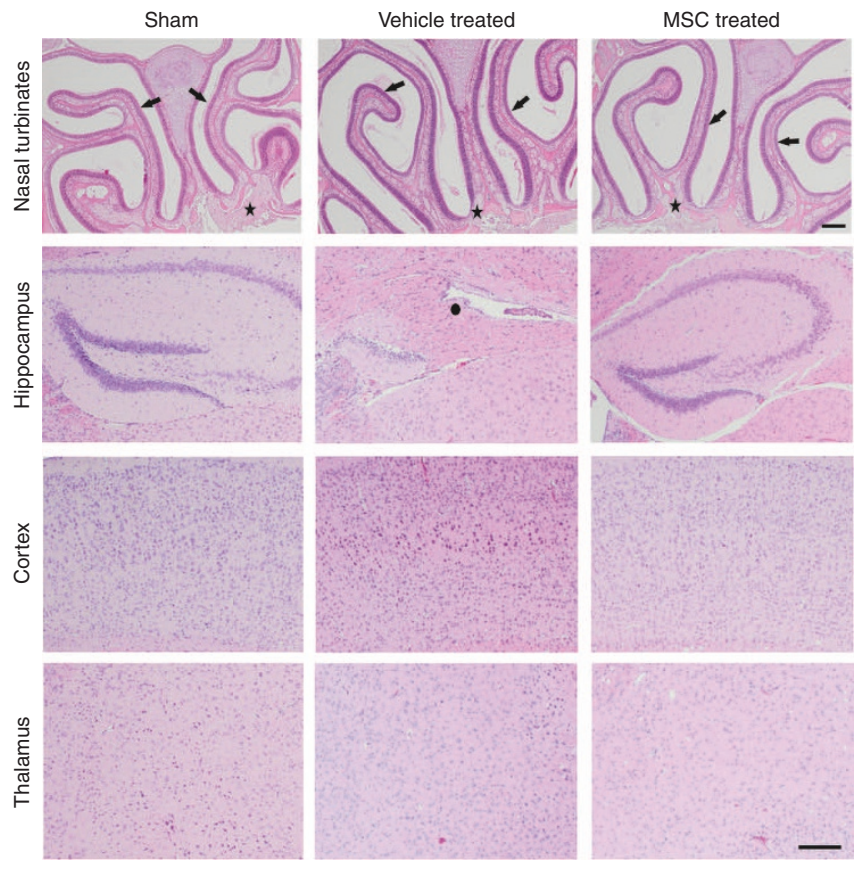

Figure 4. No malignancies are induced in the nasal turbinates or brain after intranasal MSC treatment. Hematoxylin-eosin staining of brain and nasal turbinates sections from sham-operated, HI-vehicle, and HI-MSC mice at 14 mo post-HI. No neoplasia or lesions were observed. Examples of the nasal turbinates and cortical, hippocampal, and thalamic brain regions. $n=10$ per group; arrows = nasal/olfactory mucosa; star = olfactory nerve; circle $=$ damaged hippocampus. Bar $=200 \mu \mathrm{m}$. HI, hypoxia-ischemia; MSC, mesenchymal stem cell. respectively. Our results show that at $5 \mathrm{wk}$ post-HI, the entire hippocampus and part of the sensorimotor cortex were absent following vehicle administration. MSC treatment, however, significantly decreased both gray- and white-matter damage at $5 \mathrm{wk}$ post-HI. The sensorimotor cortex was completely restored after MSC treatment. The effect of MSCs on neuronal and white matter damage remained constant over time between $5 \mathrm{wk}$ and $14 \mathrm{mo}$ (Figure 3a,b). Moreover, the sensorimotor cortex remained intact over time. In HI-vehicle mice, MAP2 area loss decreased nonsignificantly from $5 \mathrm{wk}$ to 14 mo (Figure 3a,b). In HI-vehicle mice, MBP area loss did not change significantly over time either (Figure 3a,b).

\section{MSCs Do Not Induce Neoplasia in the Brain and Nasal Turbinates in the Long Term}

To determine whether MSC treatment has any adverse longterm effects in the brain or nasal turbinates, hematoxylineosin-stained sections from sham-operated, HI-vehicle and HI-MSC mouse brains were examined for histopathological changes at 14 mo. Importantly, our results show no evidence of neoplasia in the brain or nasal turbinates in any of the examined mice ( $n=10$ per group; Figure 4 ). Several mice had mild nasal mucosal degeneration and necrosis with presence of intracellular eosinophilic hyalinized materials typical of murine nasal mucosal epithelial hyalinosis, but there were no differences between the experimental groups (sham: 2/13; HI-vehicle: 5/17; HI-MSC: 5/17). 
No Significant Increase in Systemic Pathological Lesions After MSC Treatment

Next, we determined whether intranasal MSC treatment had any adverse effects on other peripheral organs and tissues at 14 mo after $\mathrm{HI}$ induction. To this end, 38 organs per animal were analyzed for macroscopic and/or microscopic lesions. Multisystemic lymphosarcoma affecting the lymph nodes, uterus, and salivary glands was found in two mice: one HI-vehicle (1/17) and one HI-MSC (1/17) (Tables 1 and 2). One HI-MSC mouse (1/7 females) had a poorly differentiated focal uterine sarcoma that was compatible with a leiomyosarcoma. No other significant lesions were found in any of the examined mice.

\section{DISCUSSION}

Previous work by our group has demonstrated the strong therapeutic potential of intranasal MSC administration for repair of neonatal ischemic brain damage using the mouse model. Intranasal MSC treatment significantly improves motor and cognitive behavior and decreases lesion gray and white matter damage at $5 \mathrm{wk}$ post-HI. However, for translation to the clinic, it is absolutely essential to determine whether MSCs may have any adverse effects, especially during aging. Another important question is how long the positive effects of MSC-induced brain repair last. To our knowledge, this is the first study to investigate whether intranasally administered MSCs administered early in life induce tumor formation or any other microscopic or macroscopic lesions later in life. We followed the mice for a considerable period of time, as $\mathrm{C} 57 \mathrm{Bl} / 6$ mice have a normal life span of about 18-24 mo. The mice examined in our study were $>14$-mo-old when pathological assessment was carried out. We decided to terminate the experiment at this time point as $\mathrm{C} 57 \mathrm{Bl} / 6$ mice spontaneously develop tumors and other lesions around 18 mo of age, making it harder to identify the effects of MSCs. Detailed pathological analysis was performed by unbiased assessment at a pathological center. Our findings show that intranasal MSC administration does not induce any macroscopic or microscopic lesions in the brain or in any peripheral organ in the long term.

We assessed 47 mice (sham-operated $(n=13)$, HI-vehicle $(n=$ $17)$, and HI-MSC ( $n=17)$ mice) for macroscopic or microscopic lesions in the brain, nasal turbinates, and also 38 other organs
(Tables 1 and 2). All analyses were performed without prior knowledge of the mouse identification code. Our data show no evidence of tumor formation or other significant lesions in the brain of any of the examined mice (Figure 4). No tumor formation was observed in the brain or the nasal turbinates following MSC treatment. Lymphosarcoma and the other noncerebral lesions (e.g., nasal hyalinosis, lymphocytic sialoadenitis) are common findings in aged mice and are considered spontaneous lesions that are not related to intranasal MSC treatment (14). We observed lymphosarcoma following both vehicle and MSC treatment. Incidence of lymphosarcoma in B6C3F1 mice (i.e., $\mathrm{C} 57 \mathrm{BL} / 6$ and $\mathrm{C} 3 \mathrm{HJ} / \mathrm{HeJ}$ hybrids) has been reported to be $8.3 \%$ in males and $16.8 \%$ in females at the age of $22-28$ mo $(15,16)$. The uterine sarcoma observed in one HI-MSC mouse $(1 / 7$ females) is likely a primary uterine tumor that originated from the uterine smooth muscle (i.e., leiomyosarcoma) or from the uterine mesenchyme (i.e., fibrosarcoma). The finding that MSCs do not induce tumor formation or any other lesion in the brain or nasal turbinates has significant implications for the clinic.

One possible explanation as to why MSC administration has no adverse effects in the long term is that MSCs actually do not engraft in the brain following administration. In a previous study, we demonstrated that MSCs do not engraft since at $18 \mathrm{~d}$ after intracranial administration we measured $<1 \%$ of GFP-expressing MSCs by Q-PCR (13). Furthermore, we found that the number of MSCs peaks at 12 hours after intranasal treatment and decreases drastically by $72 \mathrm{~h}$ (12). On the basis of these data, we proposed that MSCs do not differentiate into neurons or astrocytes themselves but rather stimulate endogenous neural stem cells via secretion of neurotrophic factors promoting proliferation and differentiation into functional neurons $(12,17)$. Another important factor is that we administered MSCs intranasally and not systemically in our HI model, which may significantly reduce the chance of MSCs reaching peripheral tissues, thereby avoiding possible systemic adverse effects. Taken together, these data indicate that intranasal MSC administration early in life does not lead to negative adverse effects with respect to the induction of microscopic or macroscopic lesions during the life time of the animal.

As discussed before, MSCs are hardly immunogenic and have been found to possess immunosuppressive and

Table 1. Necropsy findings per animal

\begin{tabular}{lll}
\hline Sham operated & Vehicle treated & MSC treated \\
\hline Mild right corneal opacity $(n=1 / 13)$ & Mesenteric left node has lymphoma $(n=1 / 17)$ & $\begin{array}{l}\text { Multisystemic lymphoma in nodes, uterus, and } \\
\text { intestines }(n=1 / 17)\end{array}$ \\
$\begin{array}{l}\text { Right uterine mucometra/hydrometra } \\
(n=1 / 13)\end{array}$ & Acute hemorrhage: left lung lobe $(n=1 / 17)$ & $\begin{array}{l}\text { Severe cerebellar atrophy }(n=1 / 17) \\
\end{array}$ \\
& Olfactory lobe appeared smaller $(n=1 / 17)$ & Both olfactory lobes appeared atrophied $(n=1 / 17)$ \\
& Right corneal opacity $(n=1 / 17)$ & Spleen is firmer and smaller $(n=1 / 17)$ \\
& Acute linear hemorrhage in liver \\
(could be traumatic) $(n=1 / 17)$ & \\
& Liver is pale and atrophied $(n=1 / 17)$ &
\end{tabular}

Sham-operated, $n=13$; vehicle-treated, $n=17$; MSC-treated, $n=17$.

MSC, mesenchymal stem cell. 


\section{Articles $\mid$ Donega et al.}

Table 2. Full report of histopathological findings

\begin{tabular}{|c|c|c|c|}
\hline Organs & Sham operated & Vehicle treated & MSC treated \\
\hline Trachea & NSL & NSL & Mild mucosal atrophy $(n=2 / 17)$ \\
\hline Larynx & NSL & NSL & NSL \\
\hline Esophagus & NSL & NSL & NSL \\
\hline Thymus & NSL & Early lymphoma $(n=1 / 17)$ & NSL \\
\hline $\begin{array}{l}\text { Nasal turbinates (nasal mucosa, } \\
\text { olfactory mucosa and nerves) }\end{array}$ & $\begin{array}{l}\text { Mild mucosal hyalinosis } \\
(2 / 13)\end{array}$ & $\begin{array}{l}\text { Mild mucosal hyalinosis } \\
(5 / 17)\end{array}$ & $\begin{array}{l}\text { Mild mucosal hyalinosis } \\
(5 / 17)\end{array}$ \\
\hline Spinal cord, sciatic nerve & NSL & NSL & Mild gliosis in the cord $(2 / 17)$ \\
\hline Femur (bone and joint) & NSL & NSL & NSL \\
\hline Vertebra & NSL & $\begin{array}{l}\text { Minimal cartilaginous degeneration } \\
(n=2 / 17)\end{array}$ & $\begin{array}{l}\text { Minimal cartilaginous degeneration } \\
(n=2 / 17)\end{array}$ \\
\hline Bone marrow & NSL & NSL & NSL \\
\hline Heart & NSL & $\begin{array}{l}\text { Moderate lymphocytic inflammation } \\
(n=1 / 17)\end{array}$ & NSL \\
\hline \multirow[t]{2}{*}{ Salivary glands } & $\begin{array}{l}\text { Mild lymphocytic inflammation } \\
(n=8 / 13)\end{array}$ & $\begin{array}{l}\text { Mild lymphocytic inflammation } \\
(n=5 / 17)\end{array}$ & $\begin{array}{l}\text { Mild lymphocytic inflammation } \\
(n=5 / 17)\end{array}$ \\
\hline & & Early lymphoma $(n=1 / 17)$ & \\
\hline Tongue & NSL & $\begin{array}{l}\text { Mild granulomatous inflammation } \\
(n=1 / 17) \\
\text { (likely foreign body granuloma) }\end{array}$ & NSL \\
\hline Thyroid/parathyroid & NSL & NSL & NSL \\
\hline Pituitary & NSL & NSL & NSL \\
\hline \multirow[t]{3}{*}{ Liver } & $\begin{array}{l}\text { Mild biliary cystic hyperplasia } \\
(n=1 / 13)\end{array}$ & $\begin{array}{l}\text { Mild lipidosis } \\
(n=1 / 17)\end{array}$ & $\begin{array}{l}\text { Mild necrosis and inflammation } \\
(n=1 / 17)\end{array}$ \\
\hline & $\begin{array}{l}\text { Mild to moderate hepatic lipidosis } \\
(n=1 / 13)\end{array}$ & $\begin{array}{l}\text { Mild fatty change } \\
(n=1 / 17)\end{array}$ & \\
\hline & & Mild atrophy $(n=1 / 17)$ & \\
\hline Pancreas & Mild islets hyperplasia $(n=1 / 13)$ & NSL & NSL \\
\hline Testis, epididmyis & $\begin{array}{l}\text { Mild degeneration with } \\
\text { mild Leydig cell hyperplasia } \\
\text { ( } n=1 / 8 \text { males) }\end{array}$ & NSL & NSL \\
\hline Male sex glands & NSL & NSL & NSL \\
\hline Mammary gland & NSL & NSL & NSL \\
\hline Ovary & NSL & Mild to moderate atrophy $(n=2 / 10)$ & NSL \\
\hline Uterus & $\begin{array}{l}\text { Moderate pleocellular } \\
\text { inflammation ( } n=1 / 6 \text { females) }\end{array}$ & $\begin{array}{l}\text { Moderate cystic hyperplasia ( } n=1 / 10 \\
\text { females) }\end{array}$ & $\begin{array}{l}\text { Solitary leiomyosarcoma } \\
\text { ( } n=1 / 7 \text { females) }\end{array}$ \\
\hline Lung & NSL & $\begin{array}{l}\text { Early crystal pneumonia } \\
(n=1 / 17)\end{array}$ & $\begin{array}{l}\text { Mild crystal acidophilic pneumonia } \\
(n=1 / 17)\end{array}$ \\
\hline \multirow[t]{2}{*}{ Skin } & $\begin{array}{l}\text { Mild lymphocytic panniculitis } \\
(n=2 / 13)\end{array}$ & Small trichogranuloma $(n=1 / 17)$ & NSL \\
\hline & & $\begin{array}{l}\text { Mild lymphocytic and neutrophilic } \\
\text { panniculitis }(n=1 / 17)\end{array}$ & \\
\hline Skeletal muscle & NSL & $\begin{array}{l}\text { Focal necrosis with pleocellular } \\
\text { inflammation }(n=1 / 17)\end{array}$ & $\begin{array}{l}\text { Focal area of acute skeletal necrosis with } \\
\text { suppurative inflammation }(n=1 / 17)\end{array}$ \\
\hline
\end{tabular}

Sham-operated, $n=13$; vehicle-treated, $n=17$; MSC-treated, $n=17$

MSC, mesenchymal stem cell; NSL, nonsignificant lesion. 
immunomodulating capacities. For instance, MSCs secrete anti-inflammatory cytokines such as IL-10 and transforming growth factor $\beta$ and suppress expansion of $\mathrm{T}$ cells and activation of natural killer cells $(9,18,19)$. We have also shown recently that MSCs revert astrogliosis $18 \mathrm{~d}$ following HI injury (12). On the basis of these cellular characteristics, a growing number of clinical trials (www.clinicaltrials.gov) are assessing efficacy and safety to use MSCs as a therapeutic strategy for various pathologies ranging from cardiovascular to autoimmune diseases.

In this work, we show that the positive effects of intranasal MSC treatment both on sensorimotor and cognitive behavior tested by the CRT and NORT last up to 9 and 14 mo of age in neonatal mice treated with MSCs at $10 \mathrm{~d}$ post-HI. The latter is important with respect to clinical application. Our data indicate that MSC treatment results in restoration of functional tissue as HI-MSC mice still showed improved cognitive and sensorimotor performance at 9 and 14 mo after $\mathrm{HI}$ induction. Importantly, we have recently shown that treatment with human bone marrow-derived MSCs also restores motor and cognitive behavior and reduces brain infarct size in neonatal mice with HI brain injury (11). These data further strengthen the potential of using human MSCs for clinical application in neonatal brain injury in the near future.

Gray and white matter damage in HI-MSC and HI-vehicle mice did not change significantly over time, which suggests that once the lesion is formed, it does not deteriorate any further. The data in Figure 3 indicate that some endogenous repair may also take place in HI-vehicle animals as lesion size decreases (although not significantly) over time. However, we suggest on the basis of our data that the lesion is not repopulated by functionally integrated neurons in HI-vehicle mice, since we did not observe improvement in the cognitive or sensorimotor tests over time. Hence, our results demonstrate that MSC treatment provides long-term regeneration and recovery of behavioral impairments.

We show here for the first time that intranasal MSC treatment after neonatal $\mathrm{HI}$ does not induce any malignancies or other pathological abnormalities in the brain, nasal turbinates, or any peripheral tissues as shown by the unbiased and thorough assessment performed by an independent pathological center. This study also highlights the lifelong effects of intranasal MSC treatment on both cognitive and sensorimotor behavior and the brain lesion following neonatal $\mathrm{HI}$ injury in the mouse. This work may have important impact for effective and safe translation of intranasal MSC treatment to the clinic.

\section{METHODS}

\section{Ethics Statement}

Experiments were approved by the Experimental Animal Committee Utrecht (DEC-ABC, University Utrecht, Utrecht, Netherlands) and performed according to international guidelines.

\section{$\mathrm{HI}$ Induction and Intranasal MSC Administration}

An unilateral HI brain lesion was induced in 9-d-old C57BL/6 mouse pups of both sexes (Harlan Laboratories, Boxmeer, The Netherlands) under isoflurane anesthesia (induction under 5\% isoflurane; maintenance under $1.5 \%$ isoflurane in a mixture of $\mathrm{O}_{2}$ and air on a 1:1 ratio) by permanently occluding the right common carotid artery, followed by hypoxia for $45 \mathrm{~min}$ at $10 \%$ oxygen. Control mouse pups, i.e., shamoperated, underwent anesthesia and incision only. The HI procedure resulted in a mortality rate of $10 \%$. At $10 \mathrm{~d}$ post-HI, $3 \mu \mathrm{l}$ of PBS-diluted hyaluronidase (100 U; Sigma-Aldrich, St Louis, MO) was administered twice to each nostril to increase the permeability of the nasal mucosa. After $30 \mathrm{~min}$, mice received either $0.5 \times 10^{6} \mathrm{C} 57 \mathrm{BL} / 6$ mice bone marrow-derived MSCs or PBS (HI-vehicle group). Three microliters of cell suspension was administered twice to each nostril, making an end volume of $12 \mu \mathrm{l}$. Animals were randomly assigned to each experimental group, and all experimental groups were represented in all litters. Mice were provided with an igloo and running wheel as environmental enrichment from birth for the entire duration of the experiment. We did not observe any sex differences in any of the measured parameters.

In total, we used 72 animals from 17 litters. Of the 72 animals, 50 animals (sham-operated, $n=16$; HI-vehicle, $n=17$; HI-MSC, $n=17$ ) were used for long-term assessments (behavior and pathological examination) and survival up to $14 \mathrm{mo}$. However, three sham-operated animals died before the end of the study (one before day 28 and two between 6 and 9 mo after HI), leading to $n=13$ for shamoperated animals at $14 \mathrm{mo}$. In an experiment performed in parallel, an additional 22 mice were used to assess gray- and white-matter damage at $5 \mathrm{wk}$ after HI (sham-operated, $n=3$; HI-vehicle, $n=9$; HI-MSC, $n=10$ ).

\section{Mesenchymal Stem Cells}

MSCs were purchased from Invitrogen (GIBCO mouse C57BL/6 MSCs, Life Technologies, Paisley, UK) and cultured according to the manufacturer's instructions. Characterization of cell-specific antigens has been described previously by us (13). The MSCs used in this study are in agreement with the release criteria described by the International Society for Cellular Therapy, that is, more than $70 \%$ of the MSCs were positive for CD105, CD90, and CD73 and less than $10 \%$ were negative for CD45.

\section{Sensorimotor and Cognitive Function}

Unilateral sensorimotor impairment was assessed in the CRT. Weightbearing left (impaired), right (unimpaired), or both paw(s) contacting the wall during full rear were scored. Unimpaired paw preference was calculated as $(($ right - left $) /($ right + left + both $)) \times 100 \%$.

Cognitive function was assessed with the NORT. Briefly, habituation to the test environment was performed the day before the actual experiment, in an empty cage for $10 \mathrm{~min}$. At the day of the test, two similar objects were first placed in the cage, and the mouse was allowed to explore for $10 \mathrm{~min}$. The mouse was then removed from the test cage and returned to its home cage for $5 \mathrm{~min}$. In the second phase, one of the explored objects was replaced by a novel object. We measured the time that the mouse spends exploring the familiar and novel object during $5 \mathrm{~min}$. Time spent with the novel object was calculated as (interaction time novel object)/(total interaction time) $\times 100 \%$. To exclude the possibility of side bias, the familiar/novel objects were randomly allocated to either the right or left side of the cage for each animal. We did not observe any side bias as the mice showed no preference for either the left or right object during the first phase of the test (two similar objects). Also which object was designated familiar or novel was randomly assigned for each animal.

Occasionally, some animals did not perform on the behavioral tests possibly due to a lack of momentary motivation. For the CRT, our inclusion criteria were that animals should initiate at least 10 vertical explorations during $3 \mathrm{~min}$. For the NORT, animals should spend at least $30 \mathrm{~s}$ of test time ( $=10 \%$ of test time) exploring the objects. Animals that did not meet these criteria were not included in the analyses. The numbers of animals that did not perform in the CRT at 6 mo were three HI-MSC-treated animals. At 9 mo, one HI-vehicle and four HI-MSC animals did not perform in the CRT. For the NORT at 3 mo, two sham-operated, two HI-vehicle, and one HI-MSC animals did not perform. At $14 \mathrm{mo}$, five sham-operated, seven HI-vehicle, and six HI-MSC animals did not perform in the NORT. We have indicated the number of participating animals per experimental group per test in the corresponding figure legends.

\section{Pathological Assessment}

Sham-operated $(n=13)$, HI-vehicle $(n=17)$, and HI-MSC $(n=17)$ mice were submitted to the Dutch Molecular Pathology Center, Utrecht University, for post-mortem examination at 14 mo post-HI. Mice were 
killed by an overdose of pentobarbital and subsequently examined for external and internal lesions. Significant lesions were recorded, scored, and photographed. Representative specimens were collected from 39 organs (Table 2) from every mouse and fixed in 10\% neutral buffered formalin. After fixation, the organs were trimmed according to the Dutch Molecular Pathology Center standard protocol, embedded in paraffin, and sectioned. The presence of histopathological lesions was examined microscopically on hematoxylin-eosin-stained sections. The brains of 30 mice ( $n=10$ per group) were analyzed for pathological lesions by the Dutch Molecular Pathology Center, and the brains of the other animals (sham-operated, $n=3$; HI-vehicle, $n=7$; HI-MSC, $n=7$ ) were used for immunohistochemistry (see below). The skull was decalcified and sliced transversely at the level of the nasal turbinates. The sections were processed for histopathology as described above. Five brain sections and three sections from the nasal turbinates were examined for each mouse.

\section{Immunohistochemical Assessment of the Brain Lesion}

Immunohistochemical assessment of gray and white matter damage was determined in the brains of the remaining mice at 14 mo post-HI (sham-operated, $n=3$; HI-vehicle, $n=7$; HI-MSC, $n=7$ ) and at $5 \mathrm{wk}$ post-HI (sham-operated, $n=3$; HI-vehicle, $n=9$; HI-MSC, $n=10$ ). Briefly, mice were perfused intracardially with $4 \%$ paraformaldehyde, and brains were removed and embedded in paraffin. Eight micrometer coronal brain sections cut at hippocampal level were incubated with MBP (Sternberger Monoclonals, Lutherville, MD) or MAP2 (Sigma-Aldrich) followed by biotinylated horse-anti-mouse antibody (Vector Laboratories, Burliname, CA). Binding was visualized with Vectastain $\mathrm{ABC}$ kit (Vector Laboratories) and diaminobenzamidine. The percentage of ipsilateral MAP2 or MBP area loss was determined as (ipsilateral MAP2- or MBP-positive area/contralateral MAP2- or MBP-positive area) $\times 100 \%$. For both MAP2 and MBP staining, all sections were stained at the same time.

\section{Statistical Analyses}

Analyses were performed in a blinded set-up. Quantification of ipsilateral MAP2 or MBP area loss was determined by Adobe Photoshop CS5 (version $13.0 \times 64$ ) or Image J software (version 1.47; National Institute of Health), respectively. Data are presented as mean and SEM. Statistical significance was determined by using two-way ANOVA followed by Bonferroni post hoc tests for all analyses except the NORT, where statistical significance was assessed by one-sample $t$-test (two-tailed). $P<0.05$ was considered statistically significant. Outliers were detected with the Grubbs $(Q=5 \%)$ and ROUT $(Q=$ $5 \%)$ tests. Removed outliers: NORT 3 mo: one outlier (sham-operated); CRT 6 mo: three outliers (MSC-treated); CRT 9 mo: two outliers (sham-operated and MSC-treated).

\section{SUPPLEMENTARY MATERIAL}

Supplementary material is linked to the online version of the paper at http:// www.nature.com/pr

\section{ACKNOWLEDGMENTS}

The authors are greatly indebted to Sabine Versteeg, Karima Amarouchi, Elsbeth van Liere, Saskia van Essen-van Dorresteijn, and Mirjam Koster for excellent technical assistance.

\section{STATEMENT OF FINANCIAL SUPPORT}

This study was supported by the ZorgOnderzoek Nederland-Medische Wetenschappen (Zon-MW) Project (no. 11600203) and EU-7 Neurobid (HEALTH-F2-2009-241778) from the European Union.

Disclosure: The authors declare no conflicts of interest. All authors have read the journal's policy on disclosure of potential conflicts of interest.

\section{REFERENCES}

1. Dammann O, Ferriero D, Gressens P. Neonatal encephalopathy or hypoxicischemic encephalopathy? Appropriate terminology matters. Pediatr Res 2011;70:1-2.

2. de Haan M, Wyatt JS, Roth S, Vargha-Khadem F, Gadian D, Mishkin M. Brain and cognitive-behavioural development after asphyxia at term birth. Dev Sci 2006;9:350-8.

3. Ferriero DM. Neonatal brain injury. N Engl J Med 2004;351:1985-95.

4. Graham EM, Ruis KA, Hartman AL, Northington FJ, Fox HE. A systematic review of the role of intrapartum hypoxia-ischemia in the causation of neonatal encephalopathy. Am J Obstet Gynecol 2008;199: $587-95$.

5. van Handel M, Swaab H, de Vries LS, Jongmans MJ. Long-term cognitive and behavioral consequences of neonatal encephalopathy following perinatal asphyxia: a review. Eur J Pediatr 2007;166:645-54.

6. Wang CC, Chen CH, Lin WW, et al. Direct intramyocardial injection of mesenchymal stem cell sheet fragments improves cardiac functions after infarction. Cardiovasc Res 2008;77:515-24.

7. Gonzalo-Daganzo R, Regidor C, Martín-Donaire T, et al. Results of a pilot study on the use of third-party donor mesenchymal stromal cells in cord blood transplantation in adults. Cytotherapy 2009;11:278-88.

8. Deans RJ, Moseley AB. Mesenchymal stem cells: biology and potential clinical uses. Exp Hematol 2000;28:875-84.

9. Zhang R, Liu Y, Yan K, et al. Anti-inflammatory and immunomodulatory mechanisms of mesenchymal stem cell transplantation in experimental traumatic brain injury. J Neuroinflammation 2013;10:106.

10. Salem HK, Thiemermann C. Mesenchymal stromal cells: current understanding and clinical status. Stem Cells 2010;28:585-96.

11. Donega V, van Velthoven CT, Nijboer CH, et al. Intranasal mesenchymal stem cell treatment for neonatal brain damage: long-term cognitive and sensorimotor improvement. PLoS One 2013;8:e51253.

12. Donega V, Nijboer CH, van Tilborg G, Dijkhuizen RM, Kavelaars A, Heijnen CJ. Intranasally administered mesenchymal stem cells promote a regenerative niche for repair of neonatal ischemic brain injury. Exp Neurol 2014;261:53-64.

13. van Velthoven CT, Kavelaars A, van Bel F, Heijnen CJ. Mesenchymal stem cell transplantation changes the gene expression profile of the neonatal ischemic brain. Brain Behav Immun 2011;25:1342-8.

14. Taylor I. Mouse. In: McInnes EF, ed. Background Lesions in Laboratory Animals. 1st edn. Edinburgh, UK: Saunders Elsevier, 2012:45-72.

15. Ward JM, Goodman DG, Squire RA, Chu KC, Linhart MS. Neoplastic and nonneoplastic lesions in aging $(\mathrm{C} 57 \mathrm{BL} / 6 \mathrm{~N} \times \mathrm{C} 3 \mathrm{H} / \mathrm{HeN}) \mathrm{F} 1(\mathrm{~B} 6 \mathrm{C} 3 \mathrm{~F} 1)$ mice. J Natl Cancer Inst 1979;63:849-54.

16. Tarone RE, Chu KC, Ward JM. Variability in the rates of some common naturally occurring tumors in Fischer 344 rats and (C57BL/6N x C3H/ HeN)F1 (B6C3F1) mice. J Natl Cancer Inst 1981;66:1175-81.

17. van Velthoven CT, Kavelaars A, van Bel F, Heijnen CJ. Repeated mesenchymal stem cell treatment after neonatal hypoxia-ischemia has distinct effects on formation and maturation of new neurons and oligodendrocytes leading to restoration of damage, corticospinal motor tract activity, and sensorimotor function. J Neurosci 2010;30:9603-11.

18. Giuliani M, Fleury M, Vernochet A, et al. Long-lasting inhibitory effects of fetal liver mesenchymal stem cells on T-lymphocyte proliferation. PLoS One 2011;6:e19988.

19. Pluchino S, Cossetti C. How stem cells speak with host immune cells in inflammatory brain diseases. Glia 2013;61:1379-401.

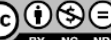

BY NC N

This work is licensed under a Creative Commons Attribution-NonCommercial-NoDerivs $\quad 4.0$ International License. The images or other third party material in this article are included in the article's Creative Commons license, unless indicated otherwise in the credit line; if the material is not included under the Creative Commons license, users will need to obtain permission from the license holder to reproduce the material. To view a copy of this license, visit http://creativecommons.org/licenses/by-nc-nd/4.0/ 